\title{
Influence of liraglutide, dulaglutide versus conventional treatment on fatty liver index and fibrosis-4 score in Egyptian patients with type 2 diabetes mellitus and non-alcoholic fatty liver disease
}

\author{
Mohammed Gameil ${ }^{1 *}$, Salah Eldeen Rozaik², Ahmed Elsebaie ${ }^{3}$, Rehab Marzouk ${ }^{4}$ \\ 1'Internal medicine dept., Endocrinology unit, Faculty of Medicine, Mansoura Univ., Mansoura, Egypt, \\ IInternal medicine dept., Gastroenterology and Hepatology unit, Faculty of Medicine, Mansoura Univ., \\ Egypt, ${ }^{3}$ Clinical pathology dept., Faculty of Medicine, Mansoura Univ., Egypt, ${ }^{4}$ Medical biochemistry dept., \\ Mansoura Univ., Egypt. \\ *drmaligameil1979@yahoo.com
}

Received: 4-9-2020

Accepted: 7-10-2020

\begin{abstract}
:
Background: Non-alcoholic fatty liver disease (NA FLD) represents a major public health challenge in patients with type 2 diabetes mellitus (T2D). It may progress to non-alcoholic steatohepatitis (NASH), cirrhosis, and carcinoma. Our aim was

independently affected by ALT changes. Conclusion: the addition of the liraglutide or dulaglutide exhibited many clinical and metabolic beneficial effects in the patients with concomitant T2D and NAFLD.
\end{abstract} to detect the changes of the fatty liver index (FLI) and FIB-4 score six months after treatment with liraglutide, or dulaglutide; novel antidiabetic medications in the Egyptian market versus the conventional therapy in Egyptian patients with T2D and co-existent NAFLD. Methods: A non-concurrent retrospective cohort study conducted at the outpatient department of authors' institution (Mansoura University hospitals) during the period from October 2019 to March 2020, included three matched groups of patients composed of Egyptian patients with T2D with age range from 35-65 years old, HbA1c level above $7.5 \%$, body mass index (BMI) above $25 \mathrm{~kg} / \mathrm{m}^{2}$, estimated glomerular filtration rate $(e G F R) \geq 60 \mathrm{~mL} / \mathrm{min} / 1.73 \mathrm{~m}^{2}$ and with concomitant NAFLD (diagnosed with hepatic ultrasonography and fatty liver index $>30$ ). Clinical history, examination with anthropometric measures, CBC, HBA1c, serum lipid, ALT, AST, GGT, serum creatinine and thyroid profile were measured before and after 24 weeks of treatment. The fatty liver index (FLI) and the FIB-4 score were used to assess hepatic steatosis and fibrosis respectively. Results: There was a significant improvement of the FIB-4 and FLI values in the patients who used liraglutide and dulaglutide compared to the conventional treatment and with better results in the liraglutide group. Moreover, there was a significant reduction of the body weight, waist circumference, BMI, SBP, DBP, GGT, ALT, AST, HBA1c, triglyceride, total cholesterol, LDL$\mathrm{C}$ and VLDL-C values in the liraglutide and dulaglutide groups versus the conventional treatment group. FLI, AST and platelet count changes were the significant predictors of the change in $\log$ FIB-4 score. However the change in log FLI was

\begin{tabular}{l}
\hline Keywords: NAFLD, Fatty liver index, FIB-4 score, \\
GLP1-RAs.
\end{tabular}

Introduction

Non-alcoholic fatty liver disease (NAFLD) represents a major public health challenge. Approximately, one-fourth of the adult population and more than half of those with type 2 diabetes mellitus (T2D) suffer from NAFLD ${ }^{1}$. It may progress to inflammation, fibrosis, and carcinoma ${ }^{2}$. Obesity, dyslipidemia, insulin resistance (IR), increased apoptosis, mitochondrial dysfunction, oxidative stress and pro-inflammatory genes induction are the most patho-logical features associating NAFLD $^{3,4}$. Clinically, It is usually under diagnosed; representing a serious clinical challenge ${ }^{5}$. Several diagnostic non-invasive methods have been used for the prediction of steatosis and fibrosis as the fatty liver index (FLI) and the FIB4 score $^{6,7}$. Glucagon-like peptide-1 receptor agonists (GLP1-RAs) are novel incretin-based licensed anti-diabetic drugs for T2D. Despite the metabolic benefits of GLP1-RAs, a lack of strong evidence of their use in treatment of NAFLD in T2D still exists $^{\mathbf{8}, 9}$. We aimed to compare the metabolic effects of the liraglutide, dulaglutide that recently introduced in the Egyptian market versus the conventional antidiabetic medications for the treatment of T2D complicated with NAFLD.

\section{Patients and Methods}

This cohort study conducted at the outpatient department of Mansoura University hospitals during the period from October 2019 to March 
2020; over 24 weeks. Our study includes three groups of diabetic patients with NAFLD with matched age and gender; group (1) control group; patients administrated the conventional treatment (metformin +/- sulfonylurea) without sodium glucose co-transporter 2 inhibitors (SGLT2i), GLP1-RAs or thiazolidinediones (TZDs) composed of 65 patients (41 male $63.1 \%$ and 24 female $36.9 \%$ ); group (2); patients administrated liraglutide (1.8 $\mathrm{mg}$ /daily) via subcutaneous injection added to their treatment, composed of 79 patients (49 male $62.0 \%$ and 30 female $38 \%$ ) and group (3) included patients administrated dulaglutide $1.5 \mathrm{mg}$ subcutaneous injection once weekly added on their treatment, composed of 80 patients (54 male $67.5 \%$ and 26 female $32.5 \%$ ). The included Egyptian patients with T2D were (35-65) years old with HbA1c level above $7.5 \%$, body mass index (BMI) above $25 \mathrm{~kg} / \mathrm{m}^{2}$, estimated glomerular filtration rate $(e G F R) \geq 60 \mathrm{~mL} / \mathrm{min} / 1.73 \mathrm{~m}^{2}$ with concomitant NAFLD (diagnosed with hepatic ultrasonography and fatty liver index >30). A written informed consent was obtained from all patients with the Institutional Review Board (IRB) approval. Detailed medical history and clinical examination with anthropometric measures were recorded in the patients' files. Following a 12-h overnight fast, venous blood was collected from each patient to estimate the glycosylated hemoglobin (HBA1c), blood lipid (serum total cholesterol [TC], triglyceride [TG], low-density lipoprotein cholesterol [LDL-C], very low-density lipoprotein cholesterol [VLDL-C] and high-density lipoprotein cholesterol [HDL-C]), complete blood count, liver enzymes (aspartate transaminase [AST], alanine transaminase [ALT], and gamma-glutamyl transferase [GGT]), renal function tests and thyroid profile before and after 24 weeks of treatment. The extent of the hepatic steatosis was estimated with the fatty liver index (FLI) ${ }^{6}$. As well as, the FIB-4 score was used to diagnose hepatic fibrosis ${ }^{7}$. Participants' follow up visits were every 8 weeks for 24 week. Adverse events were recorded on each visit and the severity assessed according to the Common Terminology Criteria for Adverse Events Version 4.0. We excluded patients with other causes of chronic liver disease like viral hepatitis, autoimmune hepatitis, history of alcohol consumption, drug-induced liver injury, biliary diseases, liver cell failure, hepatocellular carcinoma, history of diabetic ketoacidosis, concurrent administration of medications that may alter liver function tests as ( NSAIDS, statin, TZDs, etc), patients with advanced cardiac, respiratory, and/or renal dysfunction, history of GLP1-RAs hypersensitivity, intolerance, pancreatitis, history of active cancer or family history of medullary thyroid carcinoma, patients with severe gastrointestinal disease or GERD and patients with pregnancy or planning for pregnancy.

\section{Study outcome}

Our primary endpoint was the detection of the FLI and FIB-4 score changes at the end of the study period. Secondary endpoints were the detection of the significant changes in clinical and metabolic parameters between the three groups as well as the detection of any outcome variation between the liraglutide versus the dulaglutide group.

\section{Statistical analysis}

Data were analyzed using IBM SPSS Corp. Released 2013. IBM SPSS Statistics for Windows, Version 22.0. Armonk, NY: IBM Corp. Qualitative data were described using number and percent. Quantitative data were described using median with inter quartile range for non-parametric data and mean with standard deviation for parametric data after testing normality using KolmogorovSmirnov test. Significance of the obtained results was judged at the (0.05) level. Data analysis was done using Monte Carlo test for comparison of 2 or more groups of categorical variables. Student t-test \& One Way ANOVA test were used to compare parametric variables. Mann-Whitney U test \& Kruskal Wallis test were used to compare independent groups for non-parametric variables. The comparison between pre and post treatment results was tested with Paired t-test and Wilcoxon signed rank test. The Spearman's rank-order correlation is used to determine the strength and direction of a linear relationship between two non-normally distributed continuous variables and/or ordinal variables. Multiple linear regression analysis was used to detect predictors of change in FIB4 \& FLI after log transformation.

\section{Results}

Three groups of participants were included in the analysis; group (1); patients treated with conventional treatment alone, group (2); patients administrated liraglutide added on their conventional treatment and group (3); patients administrated dulaglutide added on their conventional treatment. Tables (1 \& 2) show the demographic, clinical and laboratory characters of the participants of different groups before and after 6 months of treatment. We found a significant reduction of median FIB-4 score in the liraglutide and dulaglutide groups versus the conventional treatment group ( $p$-value <0.001) with significant lower values in the liraglutide group than the dulaglutide group (p-value $=0.027$ ), tab. (2) and fig. (1). Also, a significant reduction of mean FLI was achieved in the liraglutide and 
dulaglutide groups versus the conventional treatment group ( $\mathrm{p}$-value $<0.001$ ) but without significant differences in-between the GLP1-receptor agonists groups (P- value $=0.102$ ), tab. (2) and fig. (2). GGT, ALT and AST were significantly reduced after liraglutide and dulaglutide use than the conventional treatment ( $p$-value $<0.001$ ). However, GGT was significantly lower in the liraglutide than the dulaglutide ( $\mathrm{p}$-value $<0.001$ ), tab. (2). Administration of liraglutide and dulaglutide achieved a significant HBA1c reduction than the conventional treatment (p-value <0.001) but with better results in the liraglutide group than the dulaglutide group (p- value $<0.001$ ), tab. (2).There was a significant improvement of the lipid profile (TG, TC, LDL-C, VLDL-C) in the patients administrated GLP-1 receptor agonists than the patients used the conventional treatment (p-value <0.001). VLDL-C was significantly lower in the liraglutide group than the dulaglutide group (p-value $=0.014$ ), tab. (2). SBP and DBP showed significant lower readings in the liraglutide and the dulaglutide groups than the conventional treatment group ( $\mathrm{p}$ value $<0.001$ ), with better values in the liraglutide group than the dulaglutide (p-value $<0.001$ ), tab. (1). Body weight, waist circumference and BMI were significantly reduced in the liraglutide and the dulaglutide groups than the conventional treatment group ( $\mathrm{p}$-value $<0.001$ ). However, the dulaglutide group showed better BMI values than the liraglutide group (p-value <0.001), tab. (1). With multiple linear regression analysis of our data; FLI, AST and platelet count changes were the significant predictors of the change in log FIB4 with $69.5 \%$ of FIB 4 change was predicted by the combination of these factors. ALT change was the only significant predictor of log FLI change, $\mathbf{t a b}_{\mathbf{s}}$. (3 \& 4).

Table 1. Demographic and clinical characteristics between studied groups pre and post treatment

\begin{tabular}{|c|c|c|c|c|c|c|c|}
\hline & $\begin{array}{l}\text { Group } 1 \\
\text { G1 n=65 }\end{array}$ & $\begin{array}{l}\text { Group } 2 \\
\text { G2 n=79 }\end{array}$ & $\begin{array}{l}\text { Group } 3 \\
\text { G3 n=80 }\end{array}$ & $\begin{array}{c}\text { Test of } \\
\text { significance }\end{array}$ & $\begin{array}{c}\text { G1 Vs } \\
\text { G2 }\end{array}$ & $\begin{array}{c}\text { G1 Vs } \\
\text { G } 3\end{array}$ & $\begin{array}{c}\text { G2 Vs } \\
\text { G } 3 \\
\end{array}$ \\
\hline $\begin{array}{l}\text { Age/years } \\
\text { Mean } \pm \text { SD }\end{array}$ & $51.45 \pm 6.82$ & $49.89 \pm .69$ & $51.70 \pm 7.59$ & $\mathrm{~F}=1.35 \mathrm{P}=0.26$ & 0.214 & 0.838 & 0.127 \\
\hline \multicolumn{8}{|l|}{$\operatorname{Sex} \mathbf{N}(\%)$} \\
\hline Male & $41(63.1)$ & $49(62.0)$ & $54(67.5)$ & $\mathrm{MC}$ & 0.89 & 0.58 & 0.47 \\
\hline Female & $24(36.9)$ & $30(38.0)$ & $26(32.5)$ & $\mathrm{P}=0.75$ & & & \\
\hline Duration/years & $9.0(6.0-11.0)$ & $10.0(7.0-$ & $11.0(7.25-$ & KW & $0.007 *$ & $<0.001 *$ & 0.48 \\
\hline Median (IQR) & & 16.0) & 17.75) & $\mathrm{P}=0.001^{*}$ & & & \\
\hline $\begin{array}{c}\text { Systolic blood } \\
\text { pressure } 1\end{array}$ & $151.0 \pm 10.87$ & $153.16 \pm 9.65$ & $154.63 \pm 9.70$ & $\mathrm{~F}=2.35 \mathrm{P}=0.09$ & 0.199 & $0.03 *$ & 0.36 \\
\hline \multirow[t]{2}{*}{$\begin{array}{l}\text { Systolic blood } \\
\text { pressure } 2\end{array}$} & $145.52 \pm 8.96$ & $131.58 \pm 8.15$ & $137.25 \pm 9.31$ & $\mathrm{~F}=44.7 \mathrm{P}<0.001 *$ & $<0.001^{*}$ & $<0.001 *$ & $<0.001 *$ \\
\hline & $\mathrm{P}<0.001 *$ & $\mathrm{P}<0.001 *$ & $\mathrm{P}<0.001 *$ & & & & \\
\hline $\begin{array}{l}\text { Diastolic blood } \\
\text { pressure } 1\end{array}$ & $92.92 \pm 6.67$ & $93.16 \pm 6.0$ & $95.94 \pm 6.75$ & $\mathrm{~F}=5.12 \mathrm{P}=0.0007 *$ & 0.824 & $0.006^{*}$ & $0.007 *$ \\
\hline \multirow[t]{2}{*}{$\begin{array}{l}\text { Diastolic blood } \\
\text { pressure } 2\end{array}$} & $90.92 \pm 5.29$ & $77.91 \pm 5.86$ & $81.75 \pm 8.65$ & $\mathrm{~F}=66.54 \mathrm{P}<0.001 *$ & $<0.001 *$ & $<0.001 *$ & $<0.001 *$ \\
\hline & $\mathrm{P}<0.001^{*}$ & $\mathrm{P}<0.001^{*}$ & $\mathrm{P}<0.001^{*}$ & & & & \\
\hline Weight/kg 1 & $98.96 \pm 14.33$ & $102.02 \pm 18.75$ & $101.54 \pm 15.76$ & $\mathrm{~F}=0.688 \quad \mathrm{P}=0.504$ & 0.27 & 0.35 & 0.85 \\
\hline \multirow[t]{2}{*}{ Weight / kg 2} & $104.7 \pm 14.09$ & $88.48 \pm 17.03$ & $89.35 \pm 13.31$ & $\mathrm{~F}=25.83 \mathrm{P}<0.001 *$ & $<0.001 *$ & $<0.001 *$ & 0.715 \\
\hline & $\mathrm{P}<0.001 *$ & $\mathrm{P}<0.001 *$ & $\mathrm{P}<0.001 *$ & & & & \\
\hline Height/cm & $168.02 \pm 8.74$ & $168.06 \pm 9.05$ & $169.15 \pm 8.46$ & $\mathrm{~F}=0.413 \mathrm{P}=0.66$ & 0.97 & 0.438 & 0.435 \\
\hline BMI $1\left(\mathrm{Kg} / \mathrm{m}^{2}\right)$ & $35.0 \pm 3.98$ & $36.06 \pm 5.39$ & $35.53 \pm 5.05$ & $\mathrm{~F}=0.838 \mathrm{P}=0.434$ & 0.197 & 0.518 & 0.495 \\
\hline \multirow[t]{2}{*}{ BMI $2\left(\mathrm{Kg} / \mathrm{m}^{2}\right)$} & $37.16 \pm 4.12$ & $31.26 \pm 5.09$ & $31.24 \pm 4.30$ & $\mathrm{~F}=38.91 \mathrm{P}<0.001 *$ & $<0.001 *$ & $<0.001 *$ & $<0.001 *$ \\
\hline & $\mathrm{P}<0.001 *$ & $\mathrm{P}<0.001 *$ & $\mathrm{P}<0.001 *$ & & & & \\
\hline $\begin{array}{l}\text { Waist Circum- } \\
\text { ference } 1 / \mathrm{cm}\end{array}$ & $114.78 \pm 12.86$ & $115.85 \pm 16.04$ & $116.69 \pm 13.61$ & $\mathrm{~F}=0.318 \mathrm{P}=0.728$ & 0.656 & 0.426 & 0.712 \\
\hline \multirow[t]{2}{*}{$\begin{array}{l}\text { Waist Circum- } \\
\text { ference } 2 / \mathrm{cm}\end{array}$} & $121.48 \pm 13.24$ & $102.41 \pm 14.22$ & $102.81 \pm 11.12$ & $\mathrm{~F}=49.43 \mathrm{P}<0.001 *$ & $<0.001 *$ & $<0.001 *$ & 0.847 \\
\hline & $\mathrm{P}<0.001 *$ & $\mathrm{P}<0.001^{*}$ & $\mathrm{P}<0.001 *$ & & & & \\
\hline
\end{tabular}

Group 1= control, group 2= liraglutide, group $3=$ dulaglutide. $\mathbf{1}=$ before treatment $\boldsymbol{\&} \mathbf{2}=$ after treatment . Parameters described as mean \pm standard deviation, used tests: 
Table 2. Laboratory results of the studied groups pre and post-treatment

\begin{tabular}{|c|c|c|c|c|c|c|c|}
\hline & $\begin{array}{l}\text { Group } 1 \\
\text { G1 n=65 }\end{array}$ & $\begin{array}{l}\text { Group } 2 \\
\text { G2 n=79 }\end{array}$ & $\begin{array}{l}\text { Group } 3 \\
\text { G3 n=80 }\end{array}$ & $\begin{array}{c}\text { Test of } \\
\text { significance }\end{array}$ & $\begin{array}{c}\text { G1Vs } \\
\text { G2 }\end{array}$ & $\begin{array}{l}\text { G1 Vs } \\
\text { G } 3\end{array}$ & $\begin{array}{l}\text { G2 Vs } \\
\text { G } 3\end{array}$ \\
\hline GGT1 & $91.83 \pm 16.66$ & $60.29 \pm 24.45$ & $80.73 \pm 31.29$ & $\begin{array}{l}\mathrm{F}=29.19 \\
\mathrm{P}<0.001 *\end{array}$ & $<0.001 *$ & $0.009 *$ & $<0.001 *$ \\
\hline \multirow[t]{2}{*}{ GGT2 } & $79.49 \pm 19.94$ & $26.89 \pm 13.36$ & $37.91 \pm 16.60$ & $\begin{array}{l}\mathrm{F}=193.32 \\
\mathrm{P}<0.001 *\end{array}$ & $<0.001 *$ & $<0.001^{*}$ & $<0.001 *$ \\
\hline & $\mathrm{P}<0.001 *$ & $\mathrm{P}<0.001 *$ & $\mathrm{P}<0.001^{*}$ & & & & \\
\hline TG1 & $276.85 \pm 82.24$ & $236.04 \pm 101.84$ & $275.36 \pm 163.98$ & $\begin{array}{l}F=2.68 \\
P=0.07\end{array}$ & 0.05 & 0.94 & $0.046^{*}$ \\
\hline \multirow[t]{2}{*}{ TG2 } & $224.77 \pm 90.2$ & $153.14 \pm 77.65$ & $180.21 \pm 94.93$ & $\begin{array}{l}\mathrm{F}=11.96 \\
\mathrm{P}<0.001 *\end{array}$ & $<0.001 *$ & $0.003^{*}$ & 0.053 \\
\hline & $\mathrm{P}<0.001^{*}$ & $\mathrm{P}<0.001^{*}$ & $\mathrm{P}<0.001^{*}$ & & & & \\
\hline ALT1 & $\begin{array}{c}89.0(75.5- \\
99.5)\end{array}$ & $\begin{array}{c}75.0(66.0- \\
89.0)\end{array}$ & $83.5(74.0-94.0)$ & $\begin{array}{c}\mathrm{KW} \\
\mathrm{P}<0.001 *\end{array}$ & $<0.001 *$ & 0.053 & $0.009 *$ \\
\hline \multirow[t]{2}{*}{ ALT2 } & $\begin{array}{c}\text { 78.0(64.0- } \\
92.5)\end{array}$ & $\begin{array}{c}36.0(29.0- \\
42.0)\end{array}$ & $39.5(31.0-45.0)$ & $\begin{array}{c}\mathrm{KW} \\
\mathrm{P}<0.001 *\end{array}$ & $<0.001 *$ & $<0.001 *$ & 0.072 \\
\hline & $\mathrm{P}=0.959$ & $\mathrm{P}<0.001^{*}$ & $\mathrm{P}<0.001 *$ & & & & \\
\hline AST1 & $77.25 \pm 16.99$ & $43.56 \pm 19.22$ & $53.3 \pm 23.05$ & $\begin{array}{c}\mathrm{F}=51.98 \\
\mathrm{P}<0.001^{*}\end{array}$ & $<0.001 *$ & $<0.001 *$ & $0.003 *$ \\
\hline \multirow[t]{2}{*}{ AST2 } & $57.05 \pm 14.61$ & $24.41 \pm 10.36$ & $27.55 \pm 12.1$ & $\begin{array}{l}\mathrm{F}=147.98 \\
\mathrm{P}<0.001 *\end{array}$ & $<0.001 *$ & $<0.001 *$ & 0.109 \\
\hline & $\mathrm{P}<0.001^{*}$ & $\mathrm{P}<0.001^{*}$ & $\mathrm{P}<0.001^{*}$ & & & & \\
\hline Platelet 1 & $224.88 \pm 62.52$ & $308.18 \pm 68.26$ & $302.13 \pm 66.22$ & $\begin{array}{l}\mathrm{F}=34.37 \\
\mathrm{P}<0.001^{*}\end{array}$ & $<0.001 *$ & $<0.001 *$ & 0.563 \\
\hline \multirow[t]{2}{*}{ Platelet 2} & $225.06 \pm 60.29$ & $337.37 \pm 72.93$ & $335.48 \pm 71.38$ & $\begin{array}{l}\mathrm{F}=60.22 \\
\mathrm{P}<0.001^{*}\end{array}$ & $<0.001 *$ & $<0.001 *$ & 0.863 \\
\hline & $\mathrm{P}=0.931$ & $\mathrm{P}<0.001 *$ & $\mathrm{P}<0.001^{*}$ & & & & \\
\hline TC 1 & $204.66 \pm 36.36$ & $208.61 \pm 35.27$ & $201.05 \pm 33.81$ & $\begin{array}{l}F=0.923 \\
P=0.399\end{array}$ & 0.502 & 0.538 & 0.176 \\
\hline \multirow[t]{2}{*}{ TC 2} & $199.09 \pm 39.69$ & $165.1 \pm 40.72$ & $176.53 \pm 40.59$ & $\begin{array}{l}\mathrm{F}=11.34 \\
\mathrm{P}<0.001 *\end{array}$ & $<0.001 *$ & $0.001 *$ & 0.16 \\
\hline & $\mathrm{P}=0.418$ & $\mathrm{P}<0.001 *$ & $\mathrm{P}<0.001^{*}$ & & & & \\
\hline HDL 1 & $40.03 \pm 6.88$ & $41.89 \pm 11.07$ & $39.89 \pm 11.51$ & $\begin{array}{l}F=1.27 \\
P=0.28\end{array}$ & 0.279 & 0.706 & 0.124 \\
\hline \multirow[t]{2}{*}{ HDL 2} & $42.82 \pm 10.93$ & $43.96 \pm 14.0$ & $42.33 \pm 12.76$ & $\begin{array}{c}F=0.344 \\
P=0.71\end{array}$ & 0.59 & 0.818 & 0.418 \\
\hline & $\mathrm{P}=0.11$ & $\mathrm{P}=0.062$ & $\mathrm{P}=0.015^{*}$ & & & & \\
\hline LDL 1 & $108.63 \pm 34.79$ & $117.57 \pm 33.06$ & $103.98 \pm 40.58$ & $\begin{array}{l}F=2.85 \\
P=0.06\end{array}$ & 0.144 & 0.444 & $0.019 *$ \\
\hline \multirow[t]{2}{*}{ LDL 2} & $110.23 \pm 41.16$ & $90.88 \pm 41.96$ & $93.33 \pm 40.94$ & $\begin{array}{c}\mathrm{F}=4.49 \\
\mathrm{P}=0.012 *\end{array}$ & $0.006^{*}$ & $0.015^{*}$ & 0.71 \\
\hline & $\mathrm{P}=0.816$ & $\mathrm{P}<0.001^{*}$ & $\mathrm{P}=0.02 *$ & & & & \\
\hline
\end{tabular}

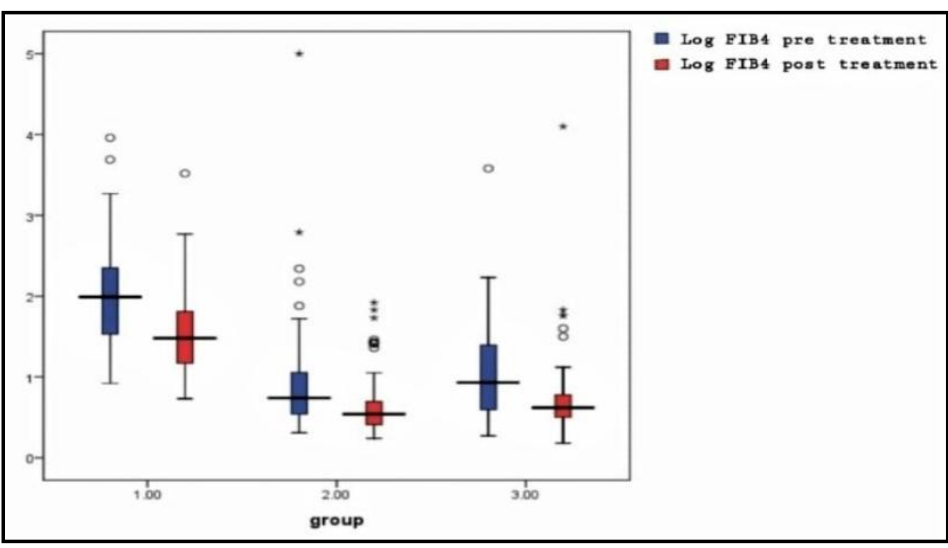

Group1(control), Group2(liraglutide), Group3(dulaglutide).

Figure 1. Log FIB-4 score changes before and after treatment in study groups. 


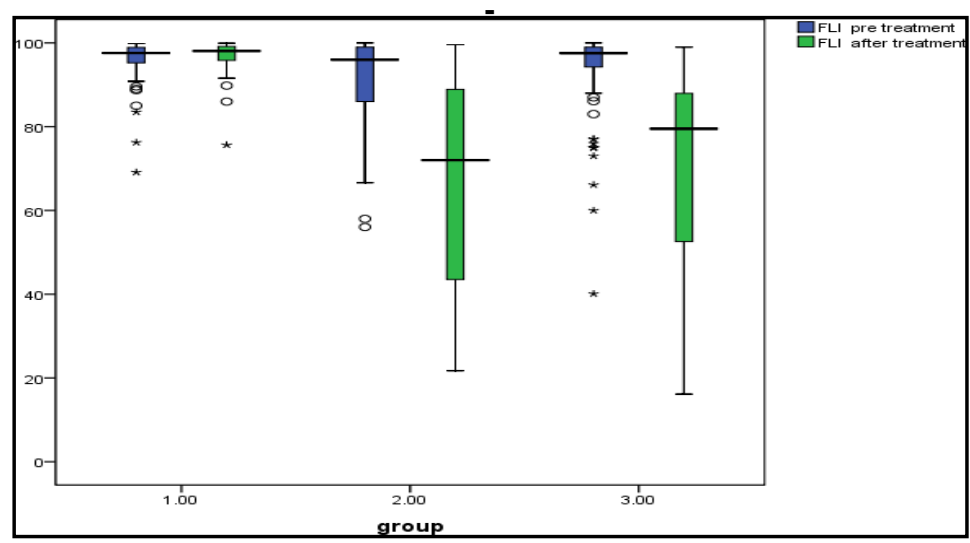

Group1 (control), Group2 (liraglutide), Group3 (dulaglutide).

Figure 2. FLI changes before and after treatment in study groups.

Table (3) Multiple linear regression for prediction of log FIB-4 changes

\begin{tabular}{lccc}
\hline Log FIB-4 & $\boldsymbol{\beta}$ & $\mathbf{t}$ & P-value \\
\hline (Constant) & & -10.953 & $<0.001^{*}$ \\
\hline FLI change & .214 & 3.343 & $<0.001^{*}$ \\
\hline AST change & -.642 & -10.640 & $<0.001^{*}$ \\
PLT change & .198 & 3.094 & $.002 *$ \\
$\mathbf{R}^{\mathbf{2}=\mathbf{0 . 6 9 5}}$ & & & \\
$\mathbf{F}=\mathbf{1 4 . 8 2}$ & & \\
$\mathbf{P}<\mathbf{0 . 0 0 1 *}$ & & & \\
\hline
\end{tabular}

Table (4) Multiple linear regression for prediction of log FLI changes.

\begin{tabular}{cccc}
\hline Log FLI & $\boldsymbol{\beta}$ & $\mathbf{t}$ & P-value \\
\hline (Constant) & & .592 & .558 \\
ALT change & -.335 & - & $.028^{*}$ \\
& & 2.303 & \\
$\mathbf{R}^{\mathbf{2}}=\mathbf{0 . 6 2 6}$ & & & \\
$\mathbf{F}=\mathbf{1 . 8 7}$ & & & \\
$\mathbf{P}=\mathbf{0 . 0 8 2}$ & & & \\
\hline
\end{tabular}

\section{Discussion}

Lifestyle modifications of diet, exercise, and weight loss represent the keystone of treatment of NAFLD and NASH. Scarce approved therapeutic options for NAFLD treatment represent a major clinical challenge particularly for patients with $\mathrm{T} 2 \mathrm{D}^{\mathbf{1 0}}$. Despite the growing promising metabolic effects of GLP1-RAs in T2D patients, the American Association for the Study of Liver Diseases (AASLD) and the National Institute for Health and Care Excellence (NICE) recommendations reported scarcity of the strong evidence to support the use of GLP-1 analogues to specifically treat liver disease in patients with NAFLD ${ }^{\mathbf{1 1}, 12}$. We found a significant reduction of median FIB-4 score and mean FLI in patients who used liraglutide and dulaglutide versus the conventional treatment. With post-hoc analysis, FIB-4 score was significantly lower in the liraglutide group than the dulaglutide group; however, FLI showed no significant difference in-between the liraglutide and dulaglutide groups. Moreover, there was a significant reduction of ALT, AST and GGT in patients who administrated liraglutide and dulaglutide versus the conventional treatment. Our findings were in accordance with Vilsbøll et al. ${ }^{13}$ who studied the effect of GLP1 receptor agonists on weight status and found a favorable effect of GLP1-RAs on overweight and liver enzymes in pre-marketing studies. Many studies demonstrated 
that, a direct action of GLP-1 receptor agonists on GLP-1 receptors located on the hepatocytes enhancing weight-loss and insulin sensitivity even in the absence of diabetes ${ }^{\mathbf{1 4}, 15}$. However, it is unclear whether the beneficial effects of GLP-1 receptor agonists are attributed to hepatic de novo lipogenesis reduction or hepatic fat oxidation increase. In vitro, GLP-1 receptors were found on hepatocytes and the direct interaction with GLP-1 receptor agonists alleviated hepatic steatosis by reducing hepatic lipogenesis as well as enhancing fatty acid consumption $^{16}$. Gupta et al. found that, GLP-1 receptor agonists use in murine NASH models led to an improvement of liver enzymes, oxidative stress, liver histology as well as weight and diabetic control $^{17}$. As well as, Fan et al. that demonstrated the insulin-sensitizing effect of liraglutide through its direct action on GLP-1 receptors expressed on hepatocytes in obesity and diabesity mice leading to a significant improvement of fasting plasma glucose levels, HOMA-IR and hepatic steatosis ${ }^{18}$. Our results in the liraglutide group agreed with Tong et al. ${ }^{19}$ who studied the effect of liraglutide on the rate of gastric emptying and found a significant weight loss with improvement of insulin sensitivity and hepatic steatosis. Also, Matikainen et al. ${ }^{\mathbf{2 0}}$ found a significant reduction of both visceral and subcutaneous adipose tissue estimated by the bioimpedance analysis (BIA) method with subsequent improvement of hepatic fat content after 16 weeks of treatment with liraglutide, however our study was of longer duration and different methodology. Also, Armstrong et al. ${ }^{21}$ studied the effect of liraglutide on insulin resistance and lipotoxicity in patients with nonalcoholic steatohepatitis (NASH) and found beneficial metabolic effects of liraglutide through decreasing de-novo lipogenesis although their study was carried out on 14 patients for 12 weeks only. Despite different methodology and study design, our findings agreed with the LEAN trial that was carried out on patients with biopsy-proven NASH, liraglutide administered subcutaneously once-daily for 48 weeks resulted in enhanced greater histological resolution of NASH and delayed fibrosis progression ${ }^{9}$. Eguchi et al. ${ }^{22}$ and Petit et al. ${ }^{23}$ studied the effect of liraglutide in different population and found a significant improvement of the NAFLD activity score, which agreed with our findings despite different methodology and ethnicity. Our findings may be attributed to the significant improvement of glycaemic control and weight reduction. However, Yang et al. attributed the liraglutide induced amelioration of hepatic steatosis in NAFLD patients to the up-regulation of the IRS2/PI3K/Akt signaling mediators which enhanced the expression and activation of insulin signaling proteins ${ }^{24}$. In contrary to, Luo et al. ${ }^{\mathbf{2 5}}$ who explained the positive effect of liraglutide on hepatic steatosis through down-regulating the expression of inflammatory and pro inflammatory cytokines, such as IL- $1 \beta$ and TNF- $\alpha$, thereby improving lipid profile and attenuating NAFLD development in diabetic mice. In contrast to our findings, Tang et al. $^{26}$ found insignificant changes of liver fat content with liraglutide. However, their study included only 35 patients for 12 weeks. In the dulaglutide group, our findings agreed with Cusi et al. ${ }^{27}$ who carried out Sub-analyses of the AWARD program (AWARD-1, AWARD-5, AWARD-8 and AWARD9) and found that once-weekly dulaglutide had significantly reduced serum ALT, AST and GGT levels compared with a placebo due to its efficacy in body weight reduction and glycaemic control. We used FLI and FIB-4 score to assess hepatis steatosis and fibrosis but Seko et al. ${ }^{28}$ used liver biopsy and fibroscan to assess liver steatosis and stiffness and found a significant improvement of liver enzymes and liver stiffness with dulaglutide that coincided with our results. Sumida et al. ${ }^{29}$ showed the cardioprotective and renoprotective effects of GLP1-RAs that may mediate a beneficial metabolic effect on hepatic steatosis. We found a significant reduction of TG, TC, LDLC, VLDL-C and HBA1c in patients used liraglutide and dulaglutide versus the conventional treatment, but the liraglutide group achieved better values of VLDL$\mathrm{C}$ and HBA1c. In accordance with Matikainen et al. ${ }^{20}$ and Marso et al. ${ }^{30}$ who attributed the cardioprotective effects of GLP-1 receptor agonists to the better glycaemic control, weight loss and plasma lipids changes. In our study, liraglutide achieved the highest effect in amelioration of SBP and DBP which were significantly reduced in the liraglutide and the dulaglutide groups versus the conventional treatment. In accordance with Zhao et al. ${ }^{31}$ and Rosso et al. ${ }^{32}$ who demonstrated the beneficial effect of liraglutide on the arterial blood pressure in individuals with or without hyperglycemia compared with placebo providing additional evidence for cardiovascular protection effect. In this study, a significant reduction of $\mathrm{BW}, \mathrm{BMI}$ and $\mathrm{WC}$ in patients who used liraglutide and dulaglutide versus the conventional treatment, but BMI was signifycantly lower in the dulaglutide group. In accordance with Inagaki et al. ${ }^{33}$ who found the addition of dulaglutide to metformin resulted in higher significant weight loss in patients with T2D. Also, Garg and Kumar reported that the use of dulaglutide as first, second, or third-line therapy in T2D management promotes weight loss with improving $\beta$ cell function, blood pressure control, serum lipid levels and cardiovascular benefits ${ }^{34}$. The major points of strength in our study were that, the use of two different GLP1-receptor agonists (liraglutide and dulaglutide) and reasonable number of participants in each arm. However, we have limitations as it was a single-center study and the ethnicity issue. 


\section{Conclusion}

GLP-1 receptor agonists exert beneficial effects on hepatic steatosis in Egyptian patients with T2D with better results after liraglutide administration. Further prospective multi-center double blinded trials with invasive histopathology and long term follow up are warranted in the future.

\author{
Abbreviations \\ Non-alcoholic fatty liver disease (NAFLD) \\ Type 2 diabetes mellitus (T2D). \\ Non-alcoholic steatohe-patitis (NASH) \\ Thiazoledendions (TZDs) \\ Glucacon like peptide receptor agonists (GLP1-RAs) \\ Sodium glucose co-transporters inhibitors (SGLT2i) \\ Body mass index (BMI) \\ Fatty liver index (FLI) \\ Fibrosis-4 index (FIB-4) \\ Insulin resistance (IR) \\ Body weight (BW) \\ Waist circumference (WC) \\ American Association for the Study of Liver Diseases \\ (AASLD)
}

National Institute for Health and Care Excellence (UK) (NICE).

\section{References}

1. Younossi Z, Koenig A, Abdelatif D, et al. Global epidemiology of nonalcoholic fatty liver diseaseMeta-analytic assessment of prevalence, incidence, and outcomes. Hepatology. 2016; 64: 73-84.

2. Chalasani N, Younossi Z, Lavine J, et al. The diagnosis and management of non-alcoholic fatty liver disease: Practice Guideline by the American Association for the Study of Liver Diseases, American College of Gastroenterology, and the American Gastroenterological Association. Hepatology. 2012; 55 (6): 2005-2023.

3. Yki-Järvinen $\mathrm{H}$. Non-alcoholic fatty liver disease as a cause and a consequence of metabolic syndrome. Lancet Diabetes Endocrinol. 2014; 2(11): 901-910.

4. Wolfs M, Gruben N, Rensen S, et al. Determining the association between adipokine expression in multiple tissues and phenotypic features of non-alcoholic fatty liver disease in obesity. Nutr Diabetes. 2015; 5 (2): e146.

5. Sanyal A, Brunt E, Kleiner D, et al. Endpoints and clinical trial design for nonalcoholic steatohepatitis. Hepatology. 2011; 54 (1): 344-353.

6. Bedogni G, Bellentani S, Miglioli L, et al. The Fatty Liver Index: A simple and accurate predictor of hepatic steatosis in the general population. BMC Gastroenterol. 2006; 6: 33.

7. Sterling R, Lissen E, Clumeck N, et al. Development of a simple noninvasive index to predict significant fibrosis patients with HIV/HCV coinfection. Hepatology. 2006; 43: 1317-1325.
8. Targher G, Bertolini L, Rodella S, et al. Nonalcoholic fatty liver disease is independently associated with an increased incidence of cardiovascular events in type 2 diabetic patients. Diabetes Care. 2007; 30: 2119-2121.

9. Armstrong M, Gaunt P, Aithal G, et al. Liraglutide safety and efficacy in patients with nonalcoholic steatohepatitis (LEAN): A multicentre, double-blind, randomised, placebo-controlled phase 2 study. Lancet. 2016; 387: 679-690.

10. Asrih M, Jornayvaz F. Diets and nonalcoholic fatty liver disease: the good and the bad. Clin Nutr. 2014; 33: 186-190.

11. National Institute for Health and Care Excellence (UK) Non-Alcoholic Fatty Liver Disease: Assessment and Management. Available from: http//www.niceorg.uklguidancelng49.

12. Chalasani N, Younossi $Z$, Lavine $J$ et al. The diagnosis and management of nonalcoholic fatty liver disease: Practice guidance from the American Association for the Study of Liver Diseases. Hepatology. 2018; 67: 328-357.

13. Vilsbøll T, Christensen M, Junker A, et al. Effects of glucagon-like peptide-1 receptor agonists on weight loss: systematic review and meta-analyses of randomised controlled trials. BMJ. 2012; 344:d7771.

14. Petit J, Verges B. GLP-1 receptor agonists in NAFLD. Diabetes Metab. 2017; 43 (1): 2S28S33.

15. Sumida Y, Yoneda M. Current and future pharmacological therapies for NAFLD/NASH. J Gastroenterol. 2018; 53 (3): 362-376.

16. Svegliati-Baroni G, Saccomanno S, Rychlicki $\mathrm{C}$, et al. Glucagon-like peptide-1 receptor activation stimulates hepatic lipid oxidation and restores hepatic signalling alteration induced by a high-fat diet in nonalcoholic steatohepatitis. Liver Int. 2011; 31: 1285-1297.

17. Gupta N, Mells J, Dunham R, et al. Glucagonlike peptide-1 receptor is present on human hepatocytes and has a direct role in decreasing hepatic steatosis in vitro by modulating elements of the insulin signaling pathway. Hepatology. 2010; 51: 1584-1592.

18. Fan R, Kang Z, He L, Chan J, Xu G, Maedler $\mathrm{K}$. Exendin-4 improves blood glucose control in both young and aging normal non-diabetic mice, possible contribution of beta cell independent effects. PLoS One 2011; 6(5):e20443.

19. Tong J, D'Alessio D. Give the receptor a brake: slowing gastric emptying by GLP-1. Diabetes. 2014; 63 (2): 407-409.

20. Niina Matikainen, Sanni Söderlund, Elias Björnson, Kirsi Pietiläinen, Antti Hakkarainen, Nina Lundbom, Marja-Riitta Taskinen, and Jan Borén. Liraglutide treatment improves postprandial lipid metabolism and cardio 
metabolic risk factors in humans with adequately controlled type 2 diabetes: A single-centre randomized controlled study. Diabetes Obes Metab. 2019; 21 (1): 84-94.

21. Armstrong M, Hull D, Guo K, et al. Glucagonlike peptide 1 decreases lipotoxicity in nonalcoholic steatohepatitis. J Hepatol. 2016; 64: 399-408.

22. Eguchi Y, Kitajima Y, Hyogo H, et al. Pilot study of liraglutide effects in non-alcoholic steatohepatitis and non-alcoholic fatty liver disease with glucose intolerance in Japanese patients (LEAN-J). Hepatol. Res. 2015; 45 : 269-278.

23. Petit J, Cercueil J, Loffroy R, et al. Effect of liraglutide therapy on liver fat content in patients with inadequately controlled type 2 diabetes: The Lira-NAFLD Study. J Clin Endocrinol Metab. 2017; 102: 407-415.

24. Pijian Y, Yuzhen L, Liraglutide ameliorates nonalcoholic fatty liver disease in diabetic mice via the IRS2/PI3K/Akt signaling pathway. Diabetes Metab Syndr Obes. 2019; 12: 10131021.

25. Ying L, Pijian Y, Zhengming L, et al. Liraglutide improves non-alcoholic fatty liver disease in diabetic mice by modulating inflammatory signaling pathways. Drug Des Devel Ther. 2019; 13: 4065-4074.

26. Tang A, Rabasa-Lhoret $\mathrm{R}$, Castel $\mathrm{H}$, et al. et al. Effects of insulin glargine and liraglutide therapy on liver fat as measured by magnetic resonance in patients with type 2 diabetes: a randomized trial. Diabetes Care. 2015; 38 : 1339-1346.

27. Cusi K, Sattar N, García-Pérez L, et al. Dulaglutide decreases plasma aminotransferases in people with Type 2 diabetes in a pattern consistent with liver fat reduction: A post hoc analysis of the AWARD programme. Diabet. Med. 2018; 35: 1434-1439.

28. Seko Y, Sumida Y, Tanaka S, et al., et al. Effect of 12-week dulaglutide therapy in Japanese patients with biopsy-proven nonalcoholic fatty liver disease and type 2 diabetes mellitus. Hepatol Res. 2017; 47: 1206-1211.

29. Yoshio S, Masashi $Y$, Katsutoshi $T$, et al. Antidiabetic therapy in the treatment of nonalcoholic steatohepatitis. Int J Mol Sci. 2020; 21 (6): 1907.

30. Marso S, Daniels G, Brown-Frandsen $K$, et al. Liraglutide and cardiovascular outcomes in type 2 diabetes. N Engl J Med. 2016; 375: 311-322.

31. Xu Z, Kun H, Meijie Z, et al. Effect of liraglutide on blood pressure: A meta-analysis of liraglutide randomized controlled trials. BMC Endocr Disord. 2019; 19: 2-14.

32. Rosso G, Labate A, Giandalia A, et al. Twelvemonth treatment with liraglutide ameliorates visceral adiposity index and common cardiovascular risk factors in type 2 diabetes outpatients. J Endocrinol Investig. 2015; 38 (1): 81-89.

33. Nobuya I, Eiichi A, Tomonori O, et al. The combination of dulaglutide and biguanide reduced bodyweight in Japanese patients with type 2 diabetes. Diabetes Obes Metab. 2016; 18 (12): 1279-1282.

34. Aakriti G, Anoop K. Risk and benefit profile of dulaglutide in established therapeutic indication. Curr Drug Saf. 2018; 13 (3): 165-170. 